\title{
DIRECT MOLECULAR DETECTION OF NUCLEIC ACIDS BY FLUORESCENCE SIGNAL AMPLIFICATION.
}

Hoang A. Ho, Kim Doré, Maurice Boissinot, Michel G. Bergeron, Robert M. Tanguay, D. Boudreau, ${ }^{*}$ and Mario Leclerc*

Department of Chemistry, the Research Center in Infectiology and the Laboratory of Cell and Developmental Genetics. Université Laval, Québec City, Qc, Canada, G1K 7P4.

\section{Supporting information}

Experiments similar to those described in figure 2 in the main text were performed on a commercial spectrofluorometer (Varian Cay Eclipse). Highly sensitive and selective detection of ss-oligonucleotides was also obtained, as shown in Figure 1. In this case, the limit of detection is about 30 copies in $3 \mathrm{~mL}$ of pure water at $55^{\circ} \mathrm{C}$.

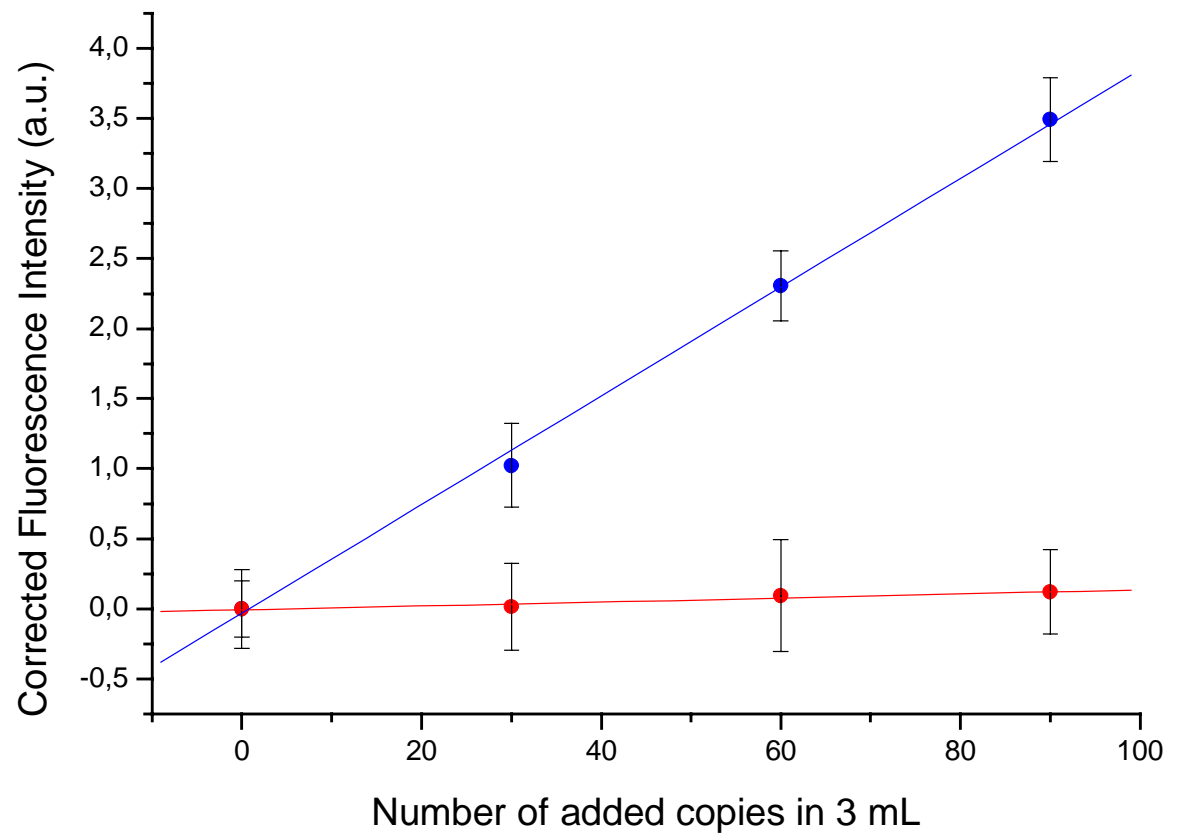

Figure 1: Corrected fluorescence intensity (after subtraction of the signal due to the initial duplex) measured at $572 \mathrm{~nm}$, with excitation at $420 \mathrm{~nm}$ in pure water at $55{ }^{0} \mathrm{C}$, as a function of the number of 20-mer oligonucleotide target copies: (blue) perfect match; (red) two mismatches. 
We also tested the applicability of the method to various kinds of genetic material, i.e. PCR products (amplicons) and human genomic DNA. Although our technique aims at eliminating the need for PCR amplification, amplicons products are an obvious choice because they are widely used in biotechnology research. Furthermore, they are also a model of double-stranded DNA, and thus serve as a good intermediate between oligonucleotides and the complete human genome. For this study, we use the probe named X1+AF546, which was also used in the oligonucleotide studies (see figure 2 in the main text), with the $C$. albicans and $C$. dubliniensis amplicons prepared as described below.

\section{Preparation of 150 bp amplicons of a portion of the EF-1a gene from $C$. albicans and $C$. dubliniensis.}

$350 \mathrm{pg}$ of genomic DNA preparation at a concentration of $350 \mathrm{pg} / \mu \mathrm{l}$ was transferred into a $19-\mu \mathrm{l}$ PCR mixture containing $50 \mathrm{mM} \mathrm{KCl}, 10 \mathrm{mM}$ Tris-HCl (pH 9.1), 0.1\% Triton X-100, $2.5 \mathrm{mM}$ $\mathrm{MgCl} 2,0.4 \mu \mathrm{M}$ concentrations of primers ECal61 [5'CAAGAAGGTTGGTTACAACCCAAAGA-3'] and Ecal184 AGGTCTTACCAGTAACTTTACCGGAT-3']), $200 \mu \mathrm{M}$ deoxynucleoside triphosphate (Amersham Biosciences, Piscataway, N.J.), $3.3 \mu \mathrm{g}$ of bovine serum albumin (Sigma-Aldrich Canada Ltd., Oakville, Ontario, Canada) per $\mu 1$, and 0.025 U of Taq DNA polymerase (Promega, Madison, Wis.) combined with the TaqStart antibody (BD Biosciences Clontech, Palo Alto, Calif.). The PCR mixtures were subjected to thermal cycling $\left(3 \mathrm{~min}\right.$ at $95^{\circ} \mathrm{C}$ and then 40 cycles of $30 \mathrm{sec}$ at $95^{\circ} \mathrm{C}$ for the denaturation step, $30 \mathrm{sec}$ at $55^{\circ} \mathrm{C}$ for the annealing step and $30 \mathrm{sec}$ at $72^{\circ} \mathrm{C}$ for the extension step) on a PTC-200 thermal cycler (MJ Research Inc., Watertown, Mass.). PCR amplification products were purified by QIAquick Gel Extraction Kit (Qiagen Inc., Mississauga, Ontario, Canada) using deionized water for eluting the amplicons. 


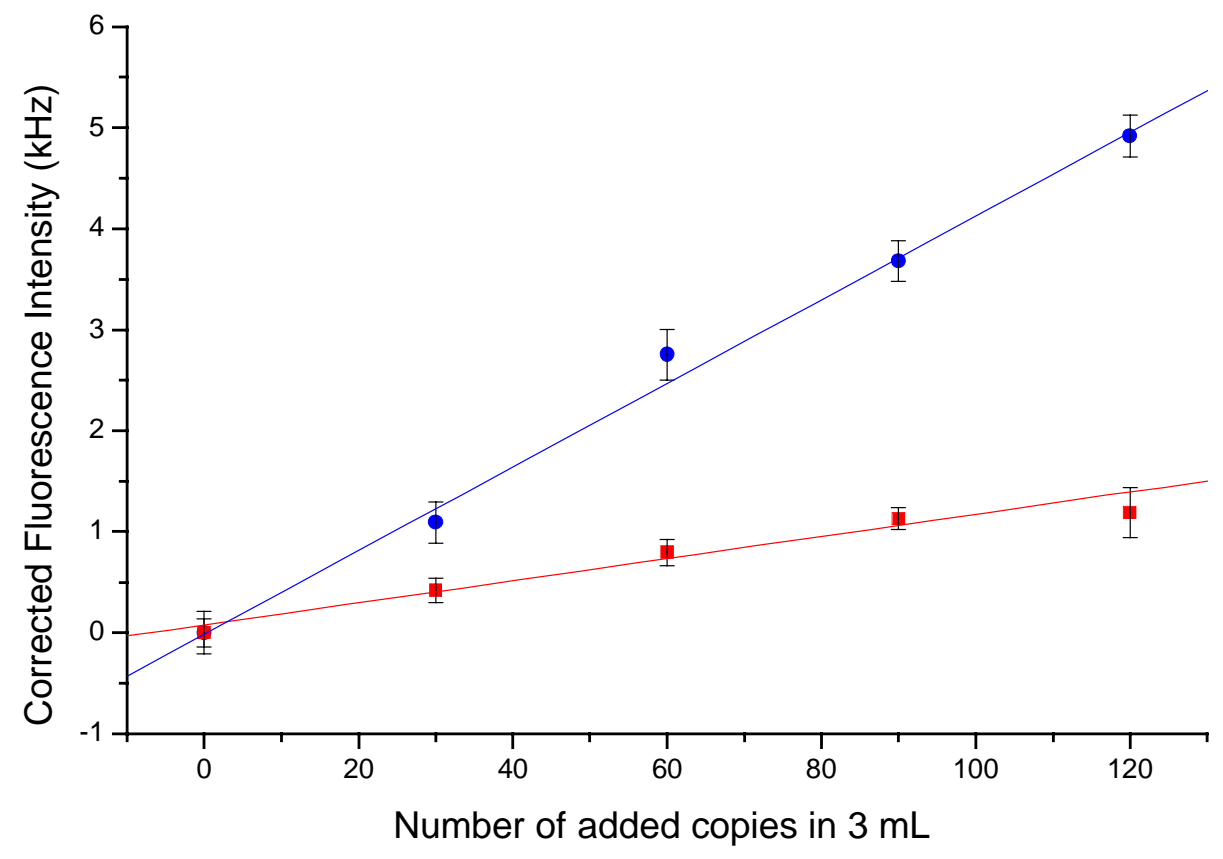

Figure 2: Corrected fluorescence intensity (after subtraction of the signal due to the initial duplex) measured at $572 \mathrm{~nm}$, with excitation at $420 \mathrm{~nm}$ in pure water at $65^{\circ} \mathrm{C}$, as a function of the number of $150 \mathrm{bp}$ amplicon target copies ; (blue) perfect match (C. albicans amplicon); (red) two mismatches (C. dubliniensis amplicon).

Figure 2 was obtained using the same procedure described in the text. One can see that the method developed can easily distinguish perfectly matched amplicons from those differing by only two nucleotides in a 150 nucleotide-long ds-DNA. This high degree of selectivity was obtained using the intrinsic signal amplification process described in the main text and optimized experimental conditions (pure water at $65^{\circ} \mathrm{C}$ ).

For human DNA, and using the same calculations and database than those reported in the experimental section of the main manuscript, we then tested our method using another probe sequence, this time specific to the E357X mutation, which is also reported as causing hereditary tyrosinemia [ref 21 in the text]. New probes specific to the E357X mutation, i.e. TAN 102a: 5'/5Alex546N/ AGGAGCCAGAAAACTTCG-3 and TAN 103a :'5'-/5Alex546N/ AGGAGCCATAAAACTTCG-3 were designed in-house and synthesised by IDT DNA 
technologies Inc. In order to test the applicability of our method to human genomic DNA extracted using various extraction techniques (in the main text we presented results obtained using the phenol-chloroform extraction method), we used human genomic DNA extracted using another, broadly used method, the Promega protocol which is described below.

\section{Purification of DNA from the blood of human healthy volunteers.} $7 \mathrm{ml}$ of blood were drawn from healthy volunteers using VacutainerTM tubes containing (K3) EDTA (Lavender caps, order number 366450, Becton Dickinson). $200 \mu \mathrm{L}$ of this blood were used for DNA extraction and purification using the MagneSil KF Genomic System (Cat no. MD1460) from Promega. The sample preparations were performed according to the manufacturer's instructions (MagneSilTM KF Genomic System, MD1460rev01) except for one slight modification: in step IVb of the Promega protocol, $200 \mu \mathrm{L}$ of TE were used instead of 200 $\mu \mathrm{l}$ of nuclease-free water. The magnetic particles were handled using a KingFisherTM mL magnetic particle processor from Thermo Labsystems driven by the "PromegaGenomic" program Version 1.0 (MagneSilTM KF genomic system, program downloaded from the Promega web site as “KfmLMagGenomv1_0”). 


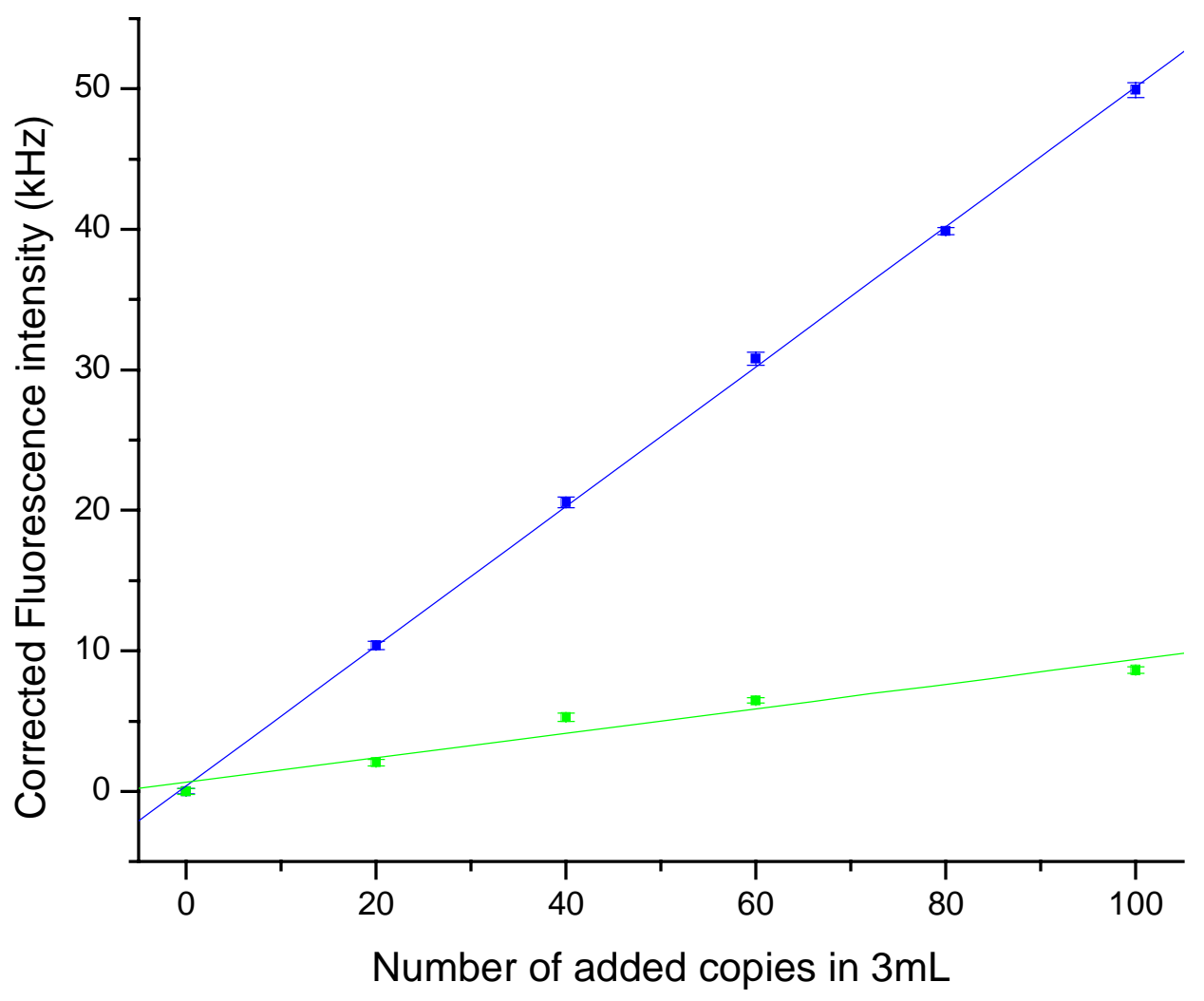

Figure 3: Corrected fluorescence intensity (after subtraction of the signal due to the initial duplex) measured at $572 \mathrm{~nm}$, with excitation at $420 \mathrm{~nm}$ in pure water at $65{ }^{\circ} \mathrm{C}$, as a function of the number of genomic DNA copies: (blue) TAN 102a + wild type genomic DNA (perfect match); (green) TAN 103a + wild type genomic DNA (one mismatch).

Figure 3 was obtained using the same experimental protocol as described in the main text. The curves obtained with the TAN102a and TAN103a probes specific to the E357X mutation are very similar to those obtained for the IVS12 mutation (Figure 4 in the main text). The higher detection sensitivity is the present case is due to the increased number of polymeric chromophoric units when working with longer, 18-mer probes (as compared with the 15-mer probes used for the IVS12 mutation). This further example of SNP detection in total, non-amplified human genomic DNA confirms the capacity of our technique to selectively and specifically detect a few copies of DNA strands in the presence of a much greater amount of non -specific DNA material. 